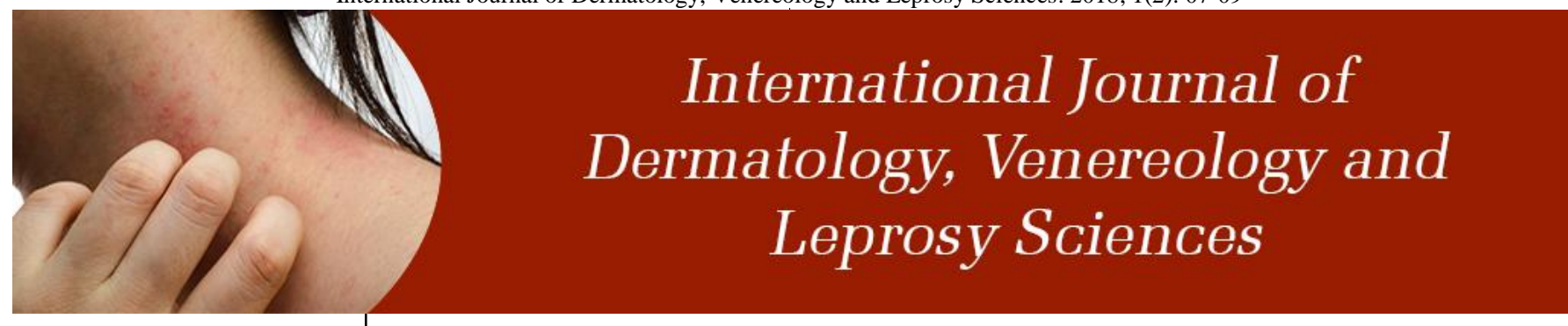

E-ISSN: 2664-942X

P-ISSN: 2664-9411

Derma 2018; 1(2): 07-09

Received: 07-05-2018

Accepted: 08-06-2018

Dr. Kavya

Department of Dermatology,

Government Dermatology

Hospital, Chevayoor,

Kozhikode, Kerala, India
Correspondence

Dr. Kavya

Department of Dermatology,

Government Dermatology

Hospital, Chevayoor,

Kozhikode, Kerala, India

\section{Evaluation of occurrence of Lichen planus pigmentosus in known population- A clinical study}

\section{Dr. Kavya}

DOI: https://doi.org/10.33545/26649411.2018.v1.i2a.11

\begin{abstract}
Background: Lichen planus pigmentosus is a rare variant of lichen planus that is seen in middle-aged individuals. The present study was conducted to determine cases of Lichen planus pigmentosus. Materials \& Methods: The present study was conducted on 108 patients of both genders. A detailed history and clinical examination was carried out, and routine hematological and biochemical tests were done.

Results: Out of 108 patients, males were 36 and females were 72. Causes of lichen planus pigmentosus was friction seen in 54, dental amalgam in 2, hair dye in 13, cosmetics in 11 and Photoaggravation in 3. The difference was significant $(P<0.05)$. Common presentation was diffuse seen in 65 , reticular in 30 , blochtcy in 10 and perifollular in 3 . The difference was significant $(P<0.05)$.

Conclusion: Causes of lichen planus pigmentosus was friction, dental amalgam, hair dye, cosmetics and Photoaggravation.
\end{abstract}

Keywords: Hematological, Lichen planus pigmentosus, Photoaggravation

\section{Introduction}

Lichen planus pigmentosus (LPP), a rare variant of lichen planus (LP), that is seen in middle-aged individuals with darker pigmented skin, was first reported in a series of Indian patients in $1974^{[1]}$. Clinically characterized by the insidious onset of focal or diffuse grayblue or dark brown macules on exposed areas, LPP is reported to have a female preponderance. Pigmentary disorders with similar clinical features have been reported in literature but it is still not fully understood as there is a lack of contemporary evidence of the published writings ${ }^{[2]}$.

Histopathological evaluation shows atrophy of the dermis with loss of rete pattern, focal basal cell vacuolization, and sparse dermal infiltrate ${ }^{[3]}$. Classically, the pattern is "actinic" with symmetric and diffuse pigmentation in sun- exposed areas, commonly in dark- skinned individuals. It can also rarely present as macular pigmentation of the flexures in the lighter- skinned. The pigmentation is dermal and occurs without any clinical evidence of inflammation ${ }^{[4]}$.

Careful examination of the BM zone is essential in distinguishing between the 2 patterns: dyskeratosis predominates in the lichenoid pattern whereas vacuolisation of the basal keratinocytes is the hallmark of vacuolar pattern ${ }^{[5]}$. A combination of both lichenoid and vacuolar interface changes may be present in certain dermatoses, such as Lupus erythematosus. The etiology of Lichen planus (LP) is unknown. Theories of infections including viral, bacterial, autoimmune, metabolic, psychosomatic and genetic causes have all had their proponents ${ }^{[6]}$. The present study was conducted to determine cases of Lichen planus pigmentosus.

\section{Materials \& Methods}

The present study was conducted in the department of Dermatology. It comprised of 108 patients of both genders. The study was approved from institutional ethical committee. All participants were informed regarding the study and written consent was obtained.

Information such as name, age, gender etc. was recorded. A detailed history and clinical examination was carried out, and routine hematological and biochemical tests were done. Results thus obtained were subjected to statistical analysis. $\mathrm{P}$ value less than 0.05 was considered significant. 


\section{Results}

Table I: Distribution of patients

\begin{tabular}{|c|c|c|}
\hline \multicolumn{3}{|c|}{ Total- 108 } \\
\hline Gender & Males & Females \\
\hline Number & 36 & 72 \\
\hline
\end{tabular}

Table I shows that out of 108 patients, males were 36 and females were 72 .

Table II: Causes of lichen planus pigmentosus

\begin{tabular}{|c|c|c|}
\hline Causes & Number & \multirow{2}{*}{ P value } \\
\hline Friction & 54 & \multirow{2}{*}{0.01} \\
\hline Dental amalgam & 2 & \\
\hline Hair Dye & 13 & \\
\hline Psychological stress & 25 & \\
\hline Cosmetics & 11 & \\
\hline Photoaggravation & 3 & \\
\hline
\end{tabular}

Table II, graph I shows that causes of lichen planus pigmentosus was friction seen in 54, dental amalgam in 2, hair dye in 13 , cosmetics in 11 and Photoaggravation in 3. The difference was significant $(P<0.05)$.

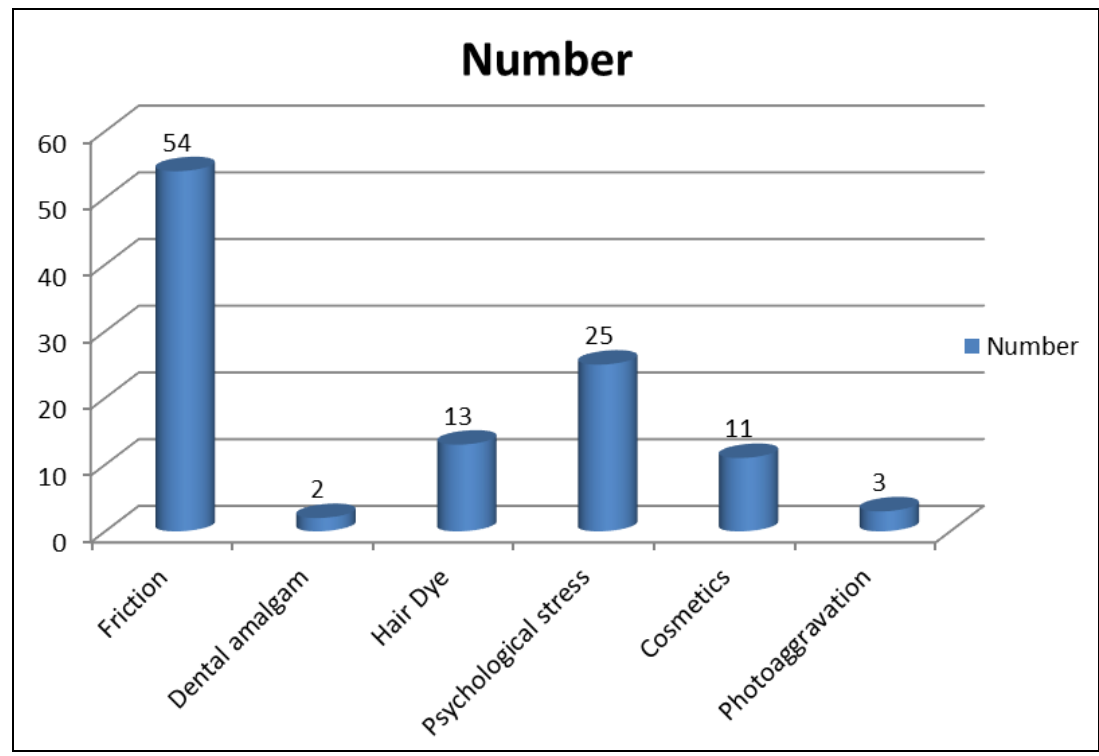

Graph I: Causes of lichen planus pigmentosus

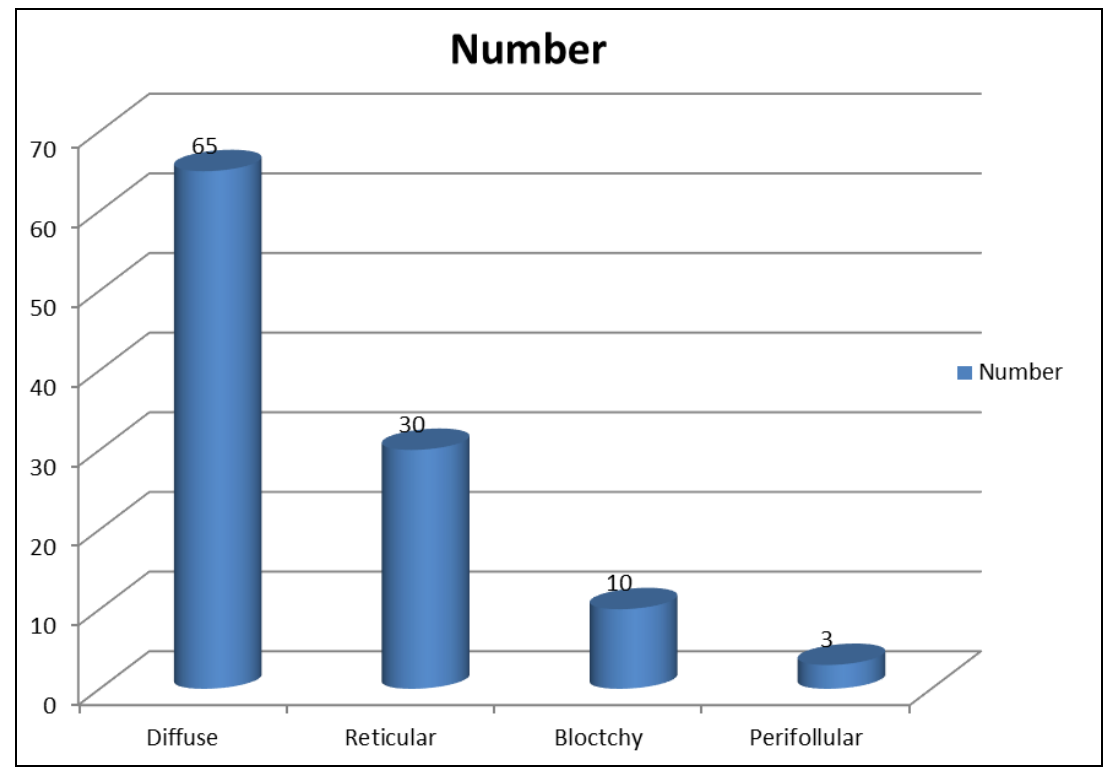

Graph II: Clinical presentation of lichen planus pigmentosus

Graph II shows that common presentation was diffuse seen

The difference was significant $(P<0.05)$. in 65 , reticular in 30 , blochtcy in 10 and perifollular in 3 . 


\section{Discussion}

LPP has a well- described association with classical lesions of LP (in about 30\%). In addition, histology of LPP is characterized by interface dermatitis with dense lichenoid infiltrate in the dermis with pigmentary incontinence ${ }^{[7]}$. Hence, it is considered a variant of LP. However, LPP has significant clinical and histological similarity with conditions such as erythema dyschromicum perstans (EDP)/ashy dermatosis (AD) and pigmented contact dermatitis, making an accurate diagnosis difficult in routine practice ${ }^{[8]}$. The pigmentation in LPP in many cases is persistent, and none of the treatment options show definite advantage over the others. The frequency of LPP varies on the basis of the population studied, with a particularly high rate of disease noted on the Indian subcontinent, most commonly affecting middle- aged people ${ }^{[9]}$. The present study was conducted to determine cases of Lichen planus pigmentosus.

In present study, out of 108 patients, males were 36 and females were 72 . We found that causes of lichen planus pigmentosus was friction seen in 54, dental amalgam in 2, hair dye in 13, cosmetics in 11 and Photoaggravation in 3.

Sassolas et al. ${ }^{[10]}$ studied histopathological sections of skin biopsies from 85 patients where after routine $\mathrm{H}$ and $\mathrm{E}$ staining and morphological changes were noted. Total numbers of biopsies inclusive of LP and LP like lesions were 85 . Among them, the number of patients having LP was 50 . The age ranged from 18 to 65 years (mean 37.1 years). The mean duration of the lesion was 12.02 months (approx 1 year). Males were affected more than females. Out of the 85 cases, 50 were Lichen planus and 35 were lichenoid eruptions. Of the Lichen planus, 31 (61.6\%) cases were of classical lichen planus, $6(13.3 \%)$ cases were of hypertrophic lichen planus, $4(8.3 \%)$ cases were of lichen planus pigmentosus, $4(6.6 \%)$ cases were of actinic lichen planus, $3(5 \%)$ cases were of eruptive lichen planus, 1 $(1.6 \%)$ case each of atrophic lichen planus, annular lichen planus.

We found that common presentation was diffuse seen in 65 , reticular in 30, blochtcy in 10 and perifollular in 3 . Bhattacharya et al. ${ }^{[11]}$ conducted a study in which skin biopsy and blood investigations were conducted and the specimens were further analyzed for their histopathological features and IHC staining for CD4+, CD8+ T- lymphocyte subsets along with CD45RO (UCHL-1), and CD68. The study showed a female preponderance (56.7\%). Photo aggravation as a precipitating cause was seen in $40 \%$ of the individuals. The lesions with duration $<4$ months had a more intense inflammatory infiltrate on histology. CD4+ and CD8+ cells showed very good Pearsons correlation on statistical analysis. CD45 was seen in association with CD8+, and staining for CD68 to assess the macrophage density showed a close correlation with CD45RO.

Recognition and diagnosis of variants require clinicopathologic correlation and the reviewing pathologist should be aware of the clinical presentation of the lesions. Lack of clinico pathologic correlation may lead to inconclusive diagnosis which mayalleviate the patient's anxiety and suboptimal treatment. Every specimen submitted should be accompanied by patient clinical information including the differential diagnosis ${ }^{[12]}$.

\section{Conclusion}

Causes of lichen planus pigmentosus was friction, dental amalgam, hair dye, cosmetics and Photoaggravation.

\section{References}

1. Bhutani LK, Bedi TR, Pandhi RK, Nayak NC. Lichen planus pigmentosus. Dermatologica. 1974; 149:43-50.

2. Kanwar AJ, Dogra S, Handa S, Parsad D, Radotra BD. A study of 124 Indian patients with lichen planus pigmentosus. Clin Exp Dermatol 2003; 28:481-5.

3. Kanwar AJ, Kaur S. Lichen planus pigmentosus. J Am Acad Dermatol 1989; 21:815.

4. Patel AB, Kubba R, Kubba A. Clinicopathological correlation of acquired hyperpigmentary disorders. Indian J Dermatol Venereol Leprol. 2013; 79:367-75.

5. Lage D, Juliano PB, Metze K, de Souza EM, Cintra ML. Lichen planus and lichenoid drug-induced eruption: A histological and immunohistochemical study. Int J Dermatol. 2012; 51:1199-205.

6. Iijima W, Ohtani H, Nakayama T, Sugawara Y, Sato E, Nagura $\mathrm{H}$ et al. Infiltrating CD8 $\mathrm{T}$ cells in oral lichen planus predominantly express CCR5 and CXCR3 and carry respective chemokine ligands RANTES/CCL5 and IP-10/CXCL10 in their cytolytic granules: A potential self-recruiting mechanism. Am J Pathol 2003; 163:261-8.

7. Lehman JS, Tollefson MM, Gibson LE. Lichen planus. Int J Dermatol. 2009; 48:682-94.

8. Hong S, Shin JH, Kang HY. Two cases of lichen planus pigmentosus presenting with a linear pattern. J Korean Med Sci. 2004; 19:152- 4.

9. Eming SA, Peters T, Hartmann K, Scharffetter Kochanek K, Mahrle G. Lichenoid chronic graft versus host disease like acrodermatitis induced by hydroxyurea. J Am Acad Dermatol. 2001; 45:321-3.

10. Sassolas B, Zagnoli A, Leroy JP, Guillet G. Lichen planus pigmentosus associated with acrokeratosis of Bazex. Clin Exp Dermatol. 1994; 19:70-3.

11. Bhattacharya M, Kaur I, Kumar B. Lichen planus: A clinical and epidemiological study. J Dermatol. 2000; 27:576-82.

12. Laskaris GC, Papavasiliou SS, Bovopoulou OD, Nicolis GD. Lichen planus pigmentosus of the oral mucosa: A rare clinical variety. Dermatologica. 1981; 162:61-3. 